\title{
Local composition fluctuations in strongly nonideal binary mixtures
}

\author{
Rajesh K. Murarka and Biman Bagchi* \\ Solid State and Structural Chemistry Unit, Indian Institute of Science, Bangalore-12, India
}

\begin{abstract}
We study the static and dynamical aspects of small length scale composition fluctuations in strongly nonideal binary mixtures of two different type by isothermal-isobaric (NPT) ensemble molecular dynamics simulations. Both the two models studied consider strong attractive interaction between dissimilar species which discourage the phase seperation in the binary mixtures. We find that the joint probability distribution of the composition fluctuations within molecular sized volumes is nearly Gaussian. However, the distributions are rather broad in both the binary mixtures. This is to be contrasted with the density fluctuation which is nearly negligible. Spontaneous fluctuation in one component is found to be anticorrelated with the fluctuations in it's counterpart. The investigation of the dynamical correlation functions of the fluctuations reveal nonexponential relaxation with a slow long time tail. The cross-correlation function relaxes at a slower rate than the two pure correlations.
\end{abstract}

\section{INTRODUCTION}

Composition fluctuations are predominant in binary liquid mixtures and play an important role both in equilibrium and dynamic behavior of such systems. The study of composition fluctuations, especially those at small length scales, should provide useful information in understanding the origin of such nonideal behavior as the nonmonotonic composition dependence of diffusion and viscosity observed in many binary mixtures. ${ }^{1}$ However, despite the importance of the composition fluctuations in the various anomalous behavior of the binary liquid mixtures, we are not aware of any explicit study on the statistical nature of local composition fluctuations and their intercorrelations in binary mixtures. The dynamical aspects of the composition fluctuations are also ill-understood.

In the recent past considerable amount of research has been devoted to the fascinating area of glass transition and the dynamics of supercooled liquids. The dynamics of the system near the glass transition temperature $\left(T_{g}\right)$ or rather in a deeply supercooled region is found to be very complex and the relaxation time of many fragile glass former show a strong non-Arrhenius temperature dependence. ${ }^{2}$ Recent time domain experiments ${ }^{3}$ carried out in various molecular glass formers have shown evidence of the spatially heterogeneous dynamics near $T_{g}$ and the length of the heterogeneous domains has been estimated to be about a few nanometer $(\sim 2-3 \mathrm{~nm})$ in size. However, one component Lennard-Jones (LJ) liquid easily crystallizes and does not form glass easily, in contrast to the binary mixtures which are known to be good glass formers. In fact, dynamical heterogeneities have also recently been observed in computer simulation studies ${ }^{4}$ of a model binary Lennard-Jones mixture. ${ }^{5}$ Furthermore, the mobility of a particle in supercooled binary Lennard-Jones mixture was observed to be related to equilibrium fluctua-

*Author to whom correspondence should be addressed. Electronic mail: bbagchi@sscu.iisc.ernet.in tions in the local composition of the mixture. ${ }^{6}$ Thus, understanding of local composition fluctuations in a binary glass forming system significantly augment our understanding of the dynamics of the system close to $T_{g}$. In particular, one would like to understand the following questions: (1) Is there any particular length scale where the fluctuations decay very slowly? (2) Can we identify two different kinds of composition fluctuations such that one is fast and the other is slow? The answer to these questions can provide a better understanding of the origin of the dynamic heterogeneity observed in the deeply supercooled liquid.

The local fluctuations which occur within a small region of system's total volume have drawn special attention in the past few years. Several interesting theoretical ${ }^{7-11}$ and computer simulation studies ${ }^{8,9,11}$ have been carried out for one component systems which show that the density fluctuations within molecular sized volumes are nearly Gaussian. In our earlier work $^{7}$ on single component supercooled liquids, which was based on classical density functional theory (DFT), it was shown that the distribution of the local equilibrium density fluctuations is nearly Gaussian and these soft localized density fluctuations (density droplets) can lead to heterogeneity in highly supercooled liquids that has been observed in experiments. ${ }^{3}$ In an important recent computer simulation study of liquid water aimed at providing a microscopic basis of hydrophobicity, Hummer et al. ${ }^{11}$ have shown that within molecular sized volumes, the distributions of density fluctuations in liquid water are nearly Gaussian. The subsequent analysis of Crooks and Chandler ${ }^{8}$ for hard sphere

TABLE I. Lennard-Jones parameters of the two different model systems considered in this study.

\begin{tabular}{lcccccc}
\hline \hline Parameters & $\sigma_{A A}$ & $\sigma_{B B}$ & $\sigma_{A B}$ & $\epsilon_{A A}$ & $\epsilon_{B B}$ & $\epsilon_{A B}$ \\
\hline $\begin{array}{l}\text { Model I } \\
\text { (equal size) }\end{array}$ & 1.0 & 1.0 & 1.0 & 1.0 & 0.5 & 2.0 \\
KA model & 1.0 & 0.88 & 0.8 & 1.0 & 0.5 & 1.5 \\
\hline \hline
\end{tabular}



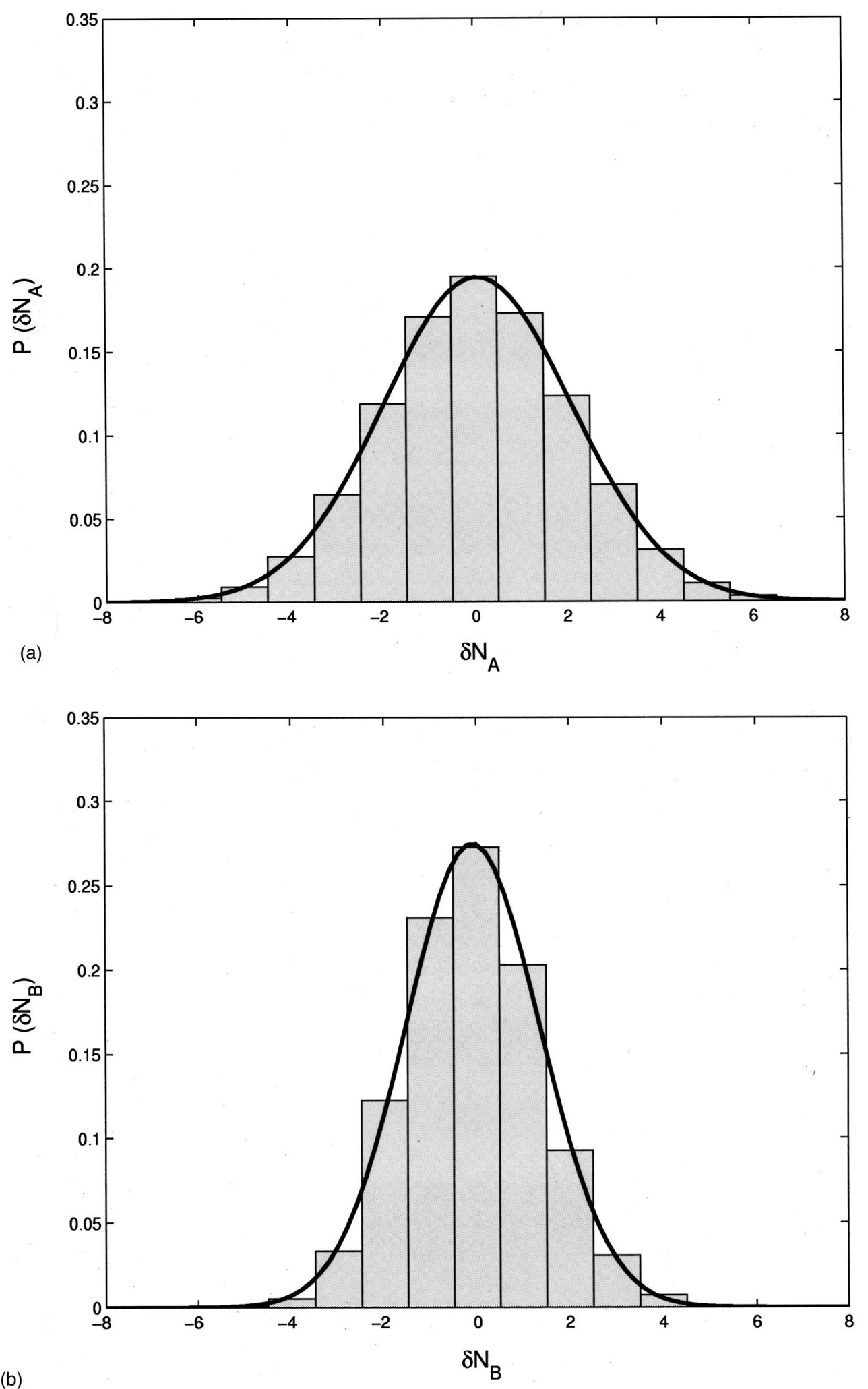

FIG. 1. The normalized probability distribution of composition fluctuations, $P\left(\delta N_{A}\right)$ and $P\left(\delta N_{B}\right)$, within a sphere of radius $R=2.0$ for equal size model (model I) at $T^{*}=1.0$ and $P^{*}=2.0$. (a) The distribution for component $A, P\left(\delta N_{A}\right)$ and (b) the same for component $B, P\left(\delta N_{B}\right)$. In both the figures, simulation results are shown by histogram and the solid line represent the Gaussian fit with the same mean and variance as these distributions. For further details, see the text.

fluid also shows that at moderate densities these distributions are very nearly Gaussian. For the Lennard-Jones fluid, recent simulation studies of Huang and Chandler ${ }^{9}$ have shown that although for large volumes and small occupation numbers the probability distributions differ from the Gaussian, for smaller volumes they are still very close to the Gaussian. The study of composition fluctuations in a localized region of a binary mixture, however, has not yet been performed. 


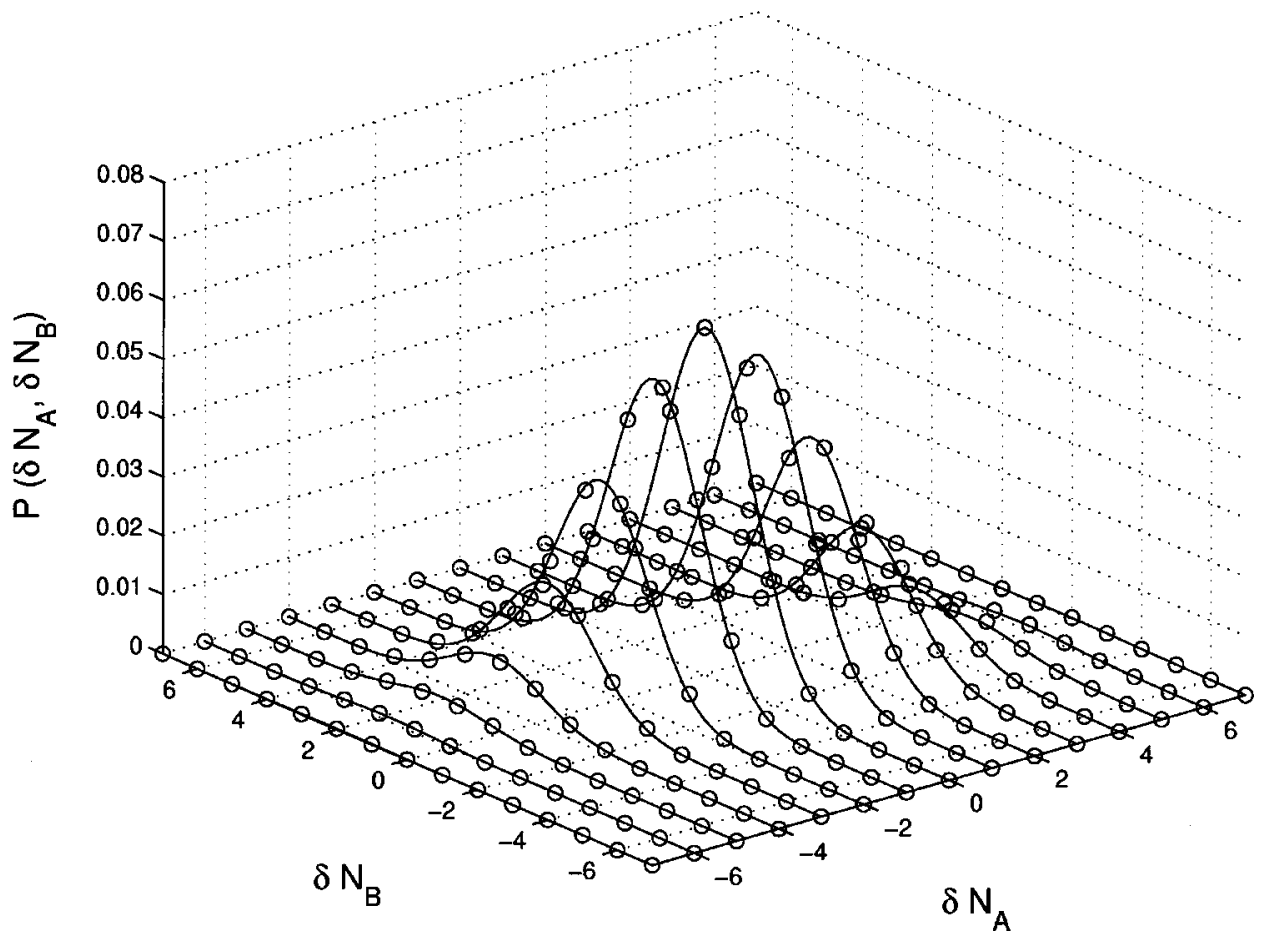

FIG. 2. The normalized joint probability distribution function $P\left(\delta N_{A}, \delta N_{B}\right)$, within a sphere of radius $R=2.0$ for the same model and at the same thermodynamic state point as is presented in Fig. 1. The open circles are the simulated results and the solid lines are the bivariate Gaussian distribution fit to the simulation results.
In this work, we present isothermal-isobaric ensemble (NPT) molecular dynamics simulation study of composition fluctuations within microscopic sized spherical volumes in two different types of model binary Lennard-Jones (LJ) mixtures. It is important to note that one should pay special attention to the segregation of the similar components while working with the binary mixtures. This problem is more serious when the interaction between the components of different kind dislike each other. Thus to avoid the possibility of the system to undergo any phase seperation, two types of model systems have been studied in this paper. Among the two models, model I (referred to as equal size model) consider the interaction strength between dissimilar species much stronger than that between the similar species, while the size and mass of both the species are same and the two species differ only in terms of the strength of the interparticle interactions. The other one (referred to as the Kob-Andersen model) has been extensively studied as a model glass former. $^{4-6,12}$ This model originally first put forward by Weber and Stillinger ${ }^{13}$ to describe amorphous $\mathrm{Ni}_{80} \mathrm{P}_{20}$ and in case of Lennard-Jones potential the subsequent parametrization was done by Kob and Andersen. ${ }^{5}$ In this model, all the interaction strength parameters and sizes are different keeping the masses of both the species same.

We find that the composition fluctuation in individual components and the joint distribution both are nearly Gaussian. Although the fluctuations are found to be quite significant in the localized regions being considered, with decrease in size of the spherical volume, the fluctuations become more predominant in view of the small number of molecules present in the smaller regions. The time autocorrelations and cross-correlation of the composition fluctuations have also been calculated. What we observe is the relaxation is nonexponential where the decay of cross-correlation function appears to be much slower. Interestingly, we find a compara- tively slower tail in the time correlation functions of fluctuation at intermediate to long time for smaller volumes. This is not surprising because in the small wave-number (k) limit, the dynamical correlations decay via well-known hydrodynamic modes in normal dense liquid states. ${ }^{14}$ However, for small volumes (that is, large k) the decay is mainly by self-diffusion in an effective potential (that is, de Gennes narrowing). ${ }^{14}$ Consequently, the slow tail observed here may be attributed to suppression of long wavelength fluctuations due to the small size of the spherical volume.

The organization of the rest of the paper is as follows. In Sec. II, we describe the details of the simulation and the two types of model system used in this study. We present the simulation results for probability distributions of composition fluctuation in Sec. III and their dynamic correlations in Sec. IV. Finally some concluding remarks are presented in Sec. VII.

\section{SYSTEM AND SIMULATION DETAILS}

We performed a series of equilibrium isothermalisobaric ensemble (NPT) molecular dynamics simulation of binary mixtures in three dimensions for a fixed value of the mole fraction of one of the species. The binary system studied here contains a total of $N=500$ particles consisting of two species of particles, $A$ and $B$ with $N_{A}=400$ and $N_{B}$ $=100$ number of $A$ and $B$ particles, respectively. Thus, the mixture consists of $80 \%$ of $A$ molecules and $20 \%$ of $B$ molecules. The interaction between any two particles is modeled by means of Lennard-Jones pair potential,

$$
u_{i j}^{\mathrm{LJ}}=4 \epsilon_{i j}\left[\left(\frac{\sigma_{i j}}{r_{i j}}\right)^{12}-\left(\frac{\sigma_{i j}}{r_{i j}}\right)^{6}\right],
$$

where $i$ and $j$ denote two different particles $(A$ and $B)$. In the 

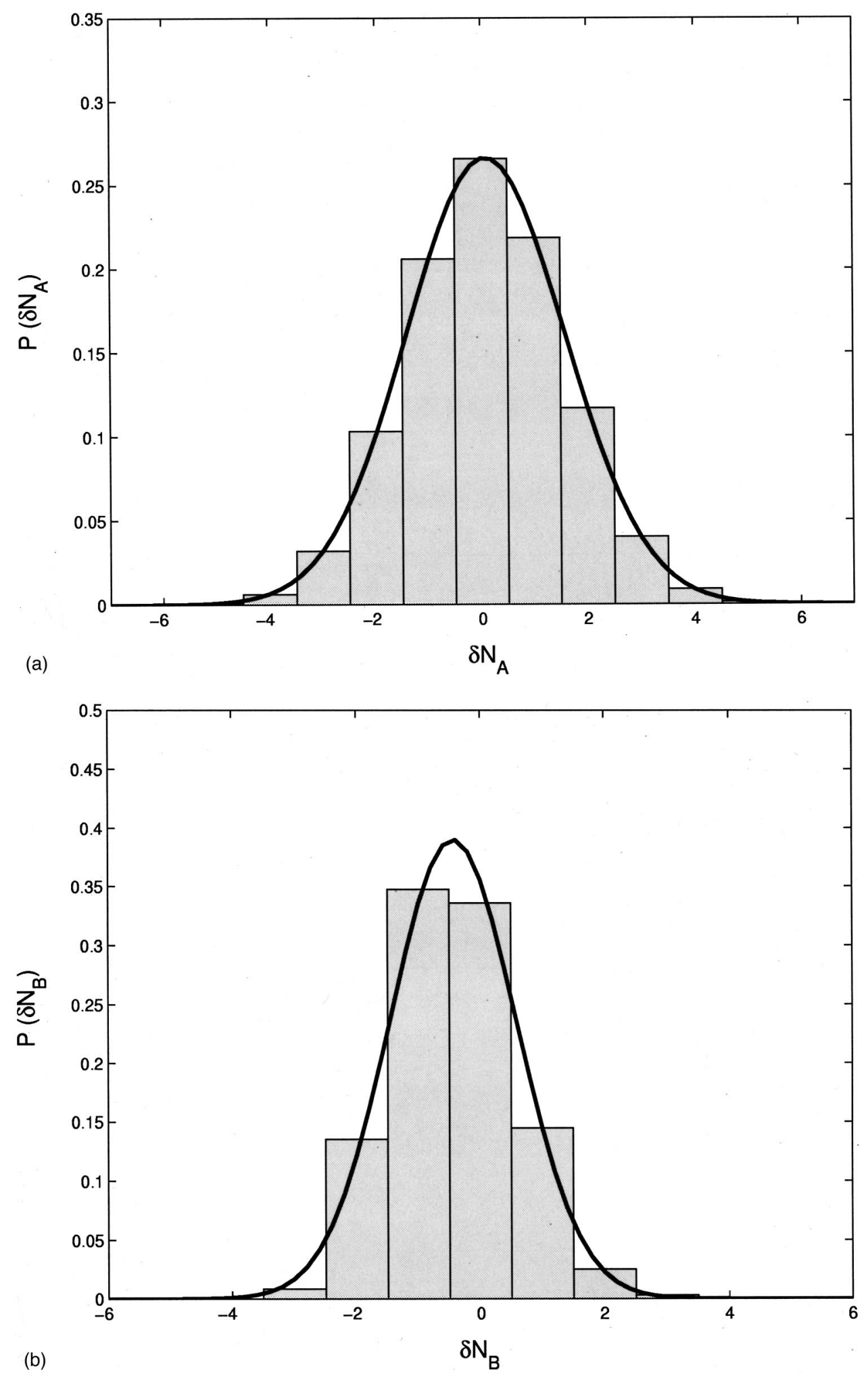

FIG. 3. Similar plot as Fig. 1 but in a smaller localized volume with $R=1.5$. (a) The fluctuation in component $A$ and (b) the fluctuation in component $B$. The histograms represent the results obtained from the simulation and the solid lines are the Gaussian distribution fit with the same mean and variance as these distributions. For details, see the text.

equal size model (model I), both types of particles $(A$ and $B)$ have the same size $\left(\sigma_{A A}=\sigma_{B B}=\sigma_{A B}=\sigma\right)$ and mass $\left(m_{A}\right.$ $\left.=m_{B}=m\right)$. The values of the Lennard-Jones interaction energy parameters $\epsilon_{i j}$ are chosen to prevent demixing and thereby to mimic the properties of the mixtures of interest.
Thus we choose $\epsilon_{A A}=1.0, \epsilon_{B B}=0.5$, whereas $\epsilon_{A B}=2.0$. It is shown elsewhere ${ }^{1}$ that this simplest model is good enough to capture the nonideality in the composition dependence of the shear viscosity. The potential parameters of the KobAndersen (KA) model $^{5}$ are as follows: $\epsilon_{A A}=1.0, \sigma_{A A}=1.0$, 

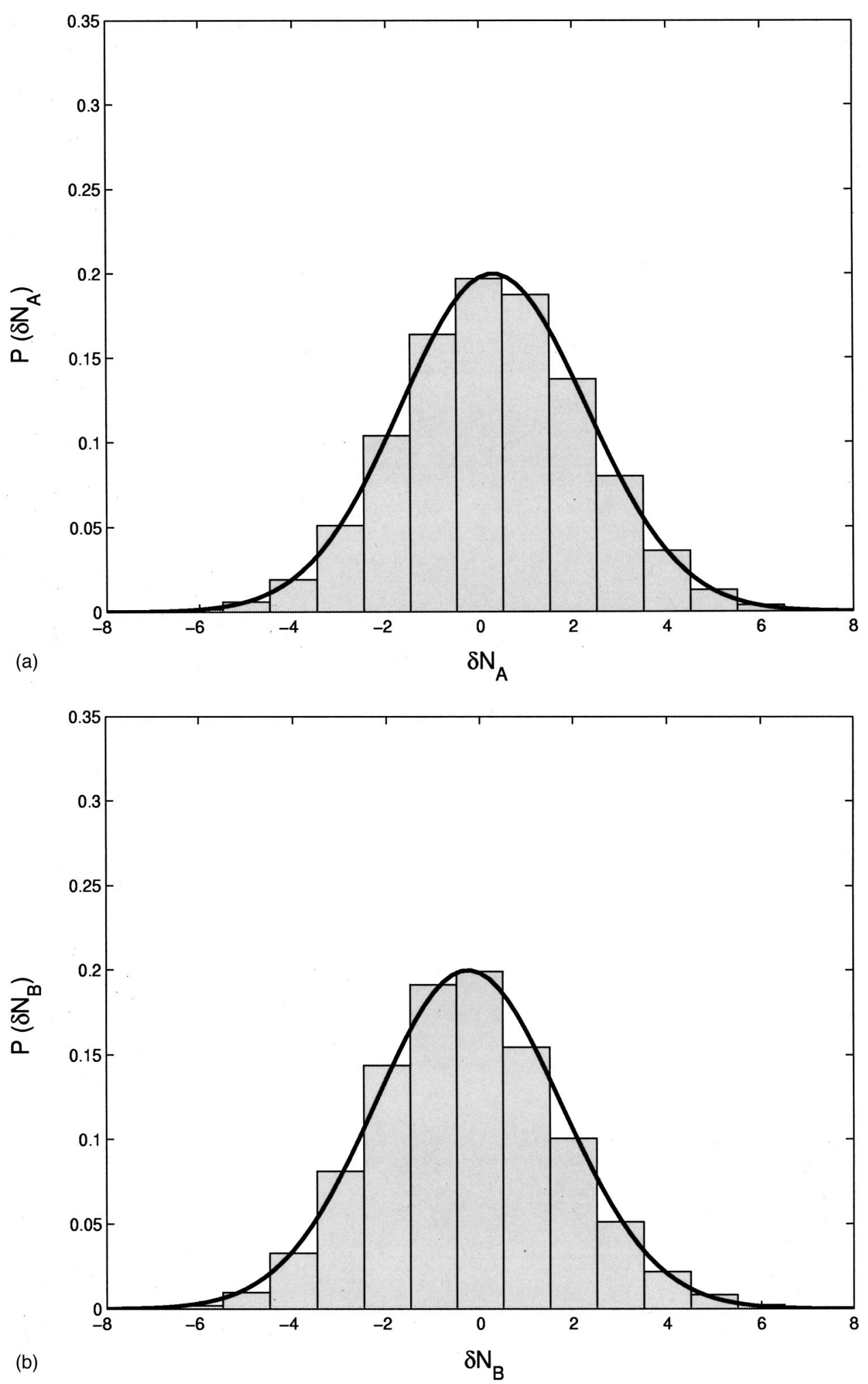

FIG. 4. The normalized probability distribution of composition fluctuations within a spherical region of radius $R=2.0$, for the Kob-Andersen model at $T^{*}=1.0$ and $P^{*}=2.0$. (a) The distribution for the component $A$. (b) Same for the component $B$. The simulation results are represented by the histograms and the solid lines are the Gaussian distribution fit with the same mean and variance as these distributions.

$\epsilon_{B B}=0.5, \sigma_{B B}=0.88, \epsilon_{A B}=1.5$, and $\sigma_{A B}=0.8$. The mass of the two species are again same $\left(m_{A}=m_{B}=m\right)$. Note that in both the two models being studied the $A B$ interaction is much stronger than both the $A A$ and $B B$ interactions. In order to lower the computational burden the potential has been truncated with a cutoff radius of $3 \sigma_{A A}$ for both type of model simulations. The usual tail corrections to the potential and the impulsive corrections of the internal pressure ${ }^{15}$ have been employed at each time step. All the quantities in this study are given in reduced units, that is, length in units of 


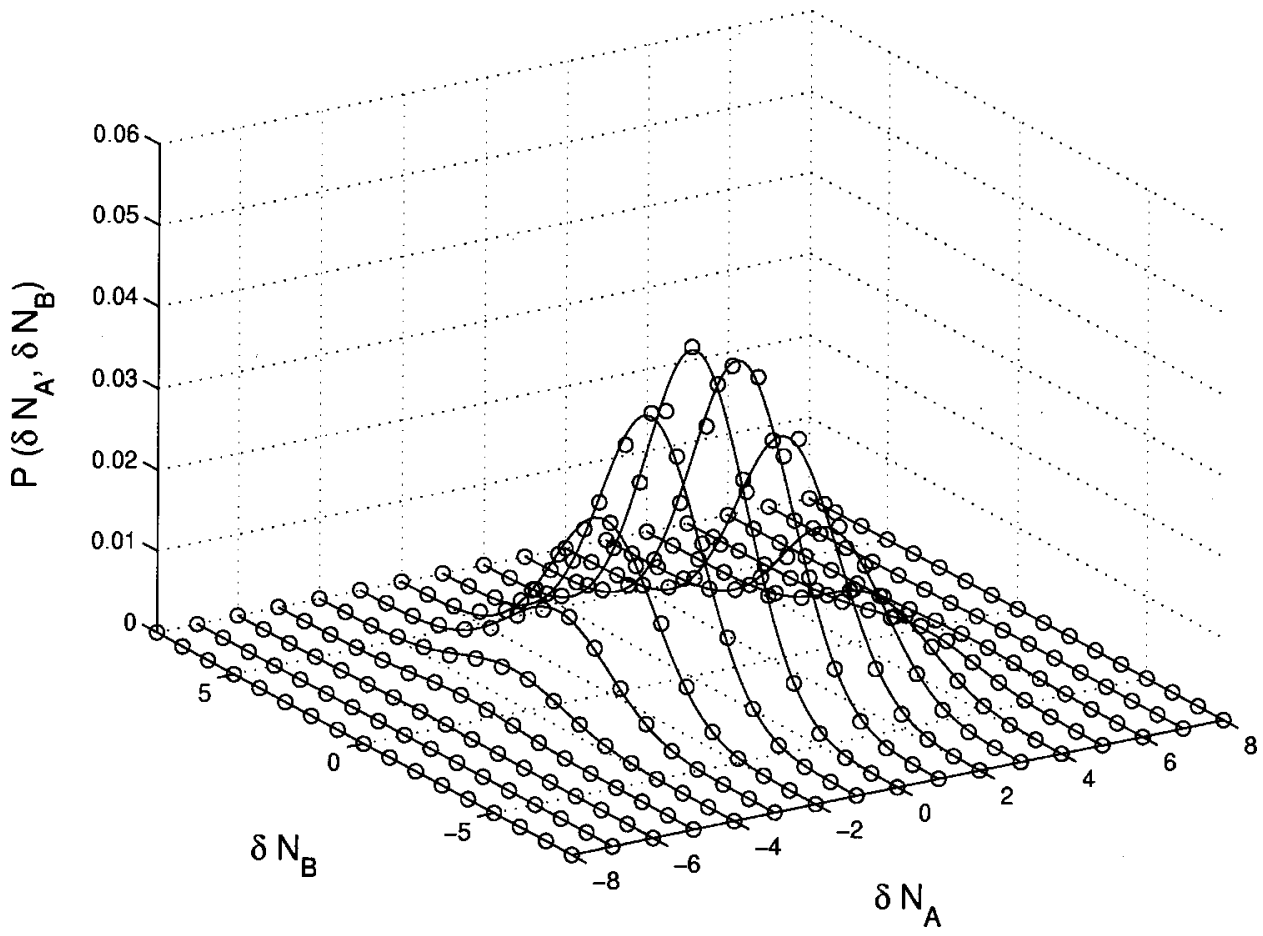

FIG. 5. The normalized joint distribution function within a spherical localized region of radius $R=2.0$ for the Kob-Andersen model at $T^{*}=1.0$ and $P^{*}=2.0$. The open circles show the simulation results while the solid lines represents the bivariate Gaussian distribution fit to the simulation results.
$\sigma_{A A}$, temperature $T$ in units of $\epsilon_{A A} / \kappa_{B}$, pressure $P$ in units of $\epsilon_{A A} / \sigma_{A A}^{3}$, and the unit of time is $\tau=\sqrt{m \sigma_{A A}^{2} / \epsilon_{A A}}$.

All simulations in the NPT ensemble were performed using the Nose-Hoover-Andersen method, ${ }^{16}$ where the external reduced temperature is held fixed at $T^{*}=1.0$. The external reduced pressure has been kept fixed at two different values $P^{*}=2.0$ and $P^{*}=4.0$. Throughout the course of the simulations, the barostat and system's degrees of freedom are coupled to an independent Nose-Hoover $\operatorname{chain}^{17}$ (NHC) of thermostats, each of length 5. The extended system equations of motion are integrated using the reversible integrator $\operatorname{method}^{18}$ with a time step of 0.002 . The higher order multiple time step method ${ }^{19}$ has been employed in the NHC evolution operator which lead to stable energy conservation for non-Hamiltonian dynamical systems. ${ }^{20}$ The extended system time scale parameter used in the calculations was taken to be 0.9274 for both the barostat and thermostats.

At each pressure, the system were equilibrated for

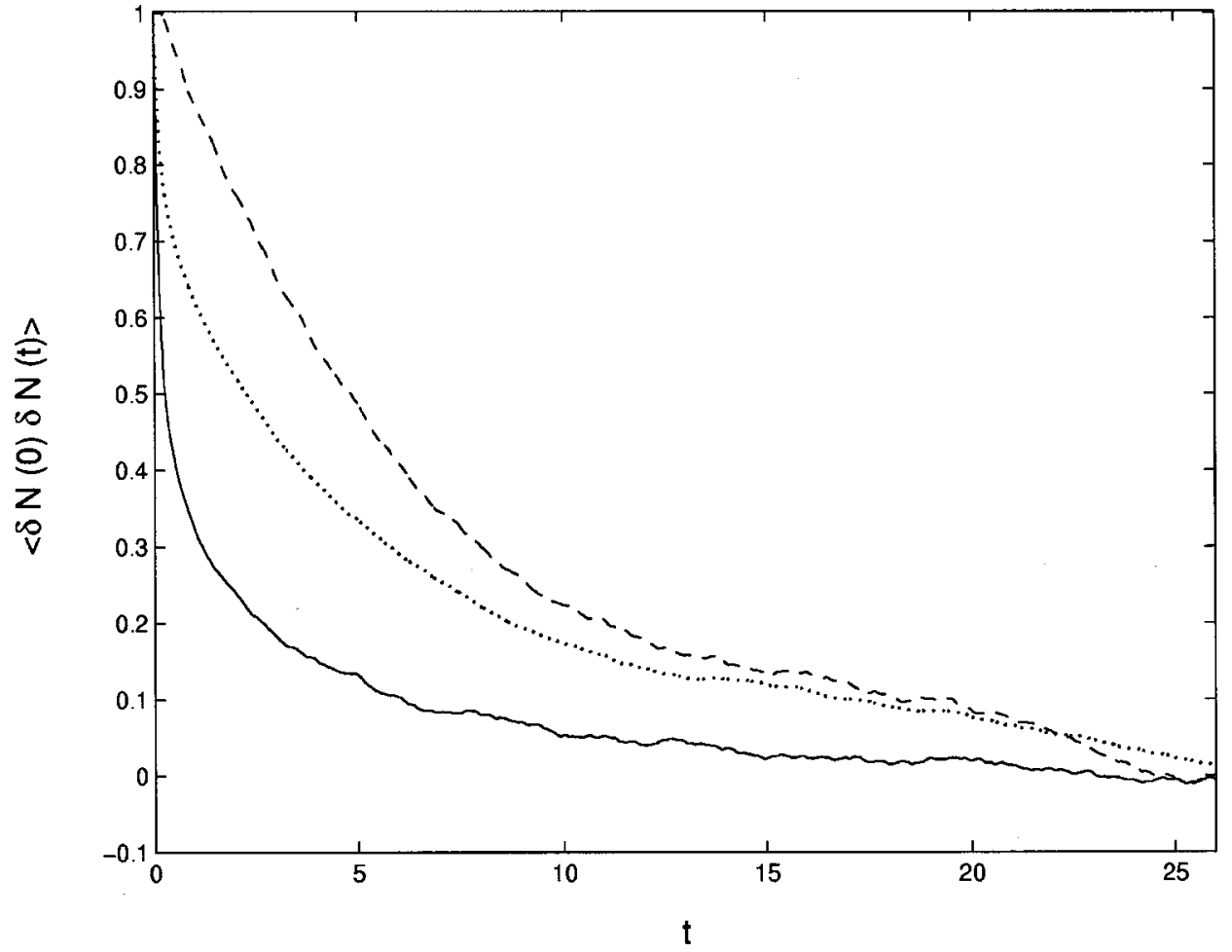

FIG. 6. The normalized time autocorrelation and cross-correlation functions of the composition fluctuations calculated within a spherical region of radius $R=2.0$ for the equal size model at $T^{*}=1.0$ and $P^{*}=2.0$. The solid line represent the $A-A$ correlation, dotted line the $B-B$ correlation, and the $A-B$ correlation shown by the dashed line. This figure shows that the relaxation of the $A-B$ correlation is the slowest one. For detailed discussion, see the text. 


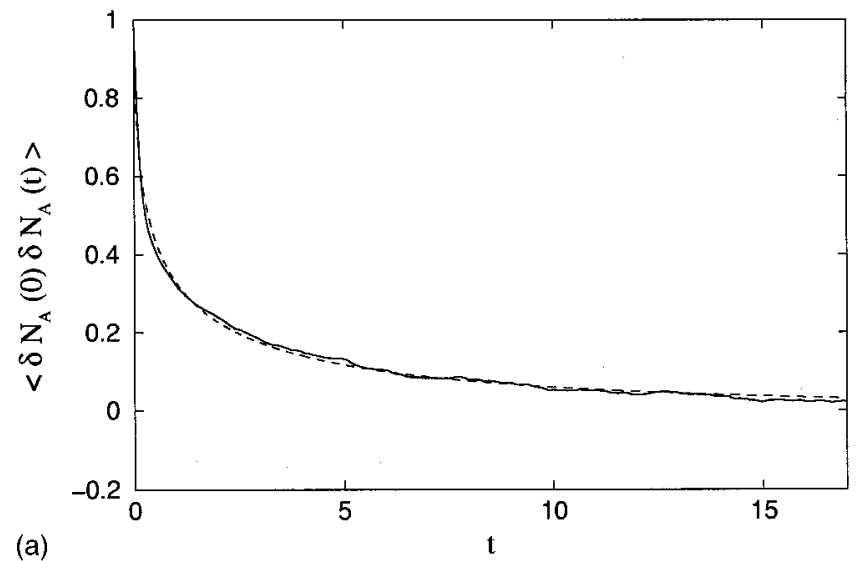

(a)
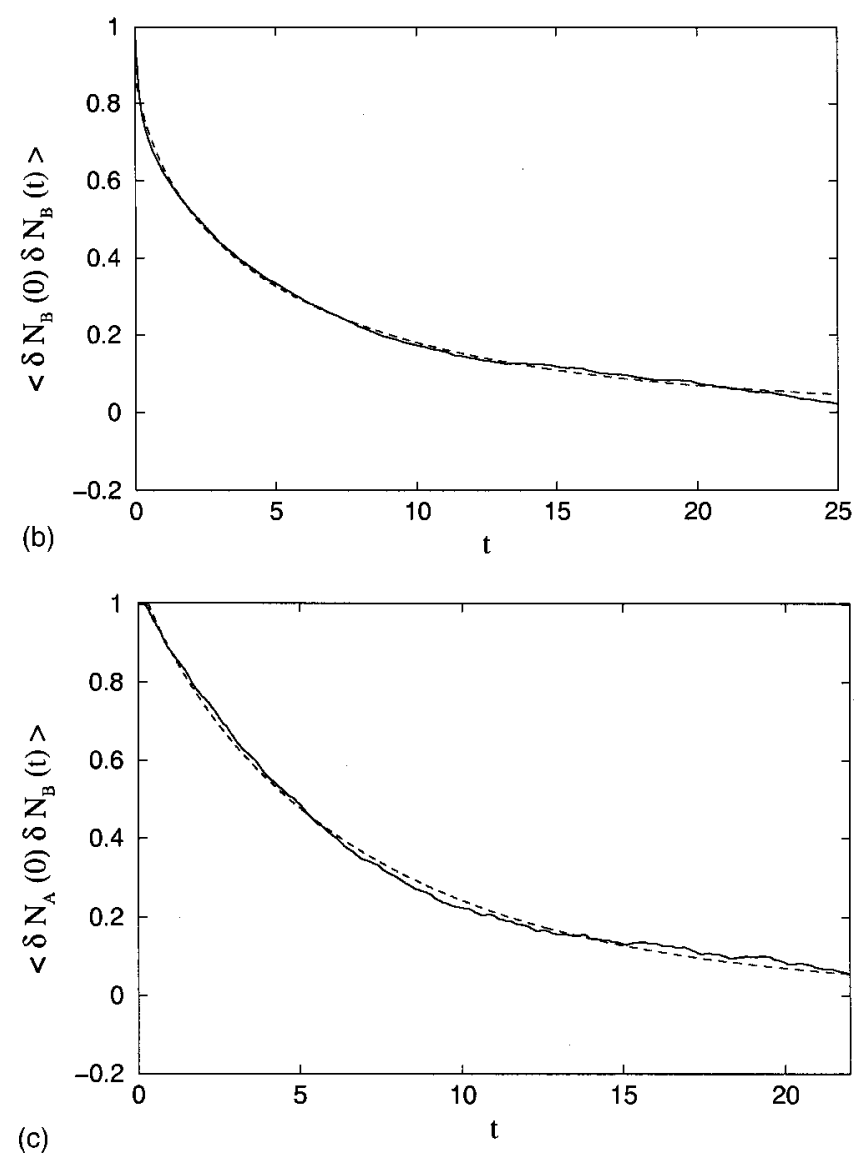

(c)

FIG. 7. The normalized time correlation functions as plotted in Fig. 6 along with the fit to a stretched exponential function. (a) For the $A-A$ correlation, (b) for the $B-B$ correlation, and (c) for the $A-B$ correlation. In each case, the solid line represent the simulation results and the dashed line the stretched exponential fit. The fitting parameters are given in Table II.

$4 \times 10^{5}$ time steps. Simulations carried out for another 4 $\times 10^{6}$ production steps following equilibration, during which the quantities of interest are calculated. We have also calculated the partial radial distribution functions in each case to make sure that the clustering or phase separation (especially among the similar species) is avoided.

The calculation of composition fluctuations has been incorporated into the simulation in the following manner. At each time step during the production stage we have computed the number of centers of each component $(A$ and $B$ ) reside in a volume $v$. After the execution of simulation, the
TABLE II. The time constant and the exponent obtained from the stretched exponential fit to the different correlations for $R=2.0$, in the equal size model.

\begin{tabular}{ccc}
\hline \hline Constants & $\tau$ & $\beta$ \\
\hline$A-A$ & 0.56 & 0.38 \\
$B-B$ & 5.04 & 0.68 \\
$A-B$ & 6.34 & 0.87 \\
\hline \hline
\end{tabular}

average number of $A$ and $B$ particles within the specified volume $v,\left\langle N_{A}\right\rangle$ and $\left\langle N_{B}\right\rangle$, respectively, are calculated. Although one could consider in general, volumes of arbitrary shape and size, for simplicity we have limited our considerations to molecular sized spherical volumes. We have considered spherical volumes of two different size with radius $R=2.0$ and $R=1.5$.

\section{SIMULATION RESULTS: EQUILIBRIUM ASPECTS}

\section{A. Probability distributions of composition fluctuation}

As mentioned earlier, both the two models studied here avoid the difficulties which can arise in studying the binary mixture due to phase seperation or crystallization. In fact, we found that indeed none of them does show any tendency of phase seperation or crystallization. Here we will present the results obtained from the simulation for the probability distributions of composition fluctuations for both the two types of model mentioned in the preceding section. The corresponding Lennard-Jones potential parameters are presented in Table I. For equal size model (model I), all simulations are performed at $P^{*}=2.0$ and at two different pressures $P^{*}$ $=2.0$ and $P^{*}=4.0$ in case of Kob-Andersen (KA) model.

\section{B. Equal size model (model I)}

Figure 1 shows the normalized probability distributions of the composition fluctuation for both the components in the spherical volume of radius $R=2.0$. For the thermodynamic state point $\left(P^{*}=2.0\right.$ and $\left.T^{*}=1.0\right)$, the reduced average density $\bar{\rho}^{*}$ of the system is 0.894 . Figure 1 (a) shows the composition fluctuation of component $A$, while that of $B$ is shown in Fig. 1(b). In both the figures simulation results are shown by the histograms. For reference, the solid lines represent the pure Gaussians with the same mean and variance as these distributions. As can be seen from these figures, the probability distributions for the composition fluctuation are nearly Gaussian, centered around the average value of the respective components in both the cases. In Fig. 1, the average number of components $A$ and $B$ are $\left\langle N_{A}\right\rangle=24.06$ and $\left\langle N_{B}\right\rangle$ $=5.91$, respectively. The spread of the distribution becomes broader on going from the minority species $(B)$ to the majority species $(A)$ [as can be seen from the Figs. 1(a) and 1(b)]. The respective standard deviations for $A$ and $B$ are 2.05 and 1.45 . Figure 1 clearly demonstrate that the local number density fluctuations of both the species are rather large. This could be the reason for the nonideality in the composition dependence of viscosity in binary mixtures. ${ }^{1}$ 


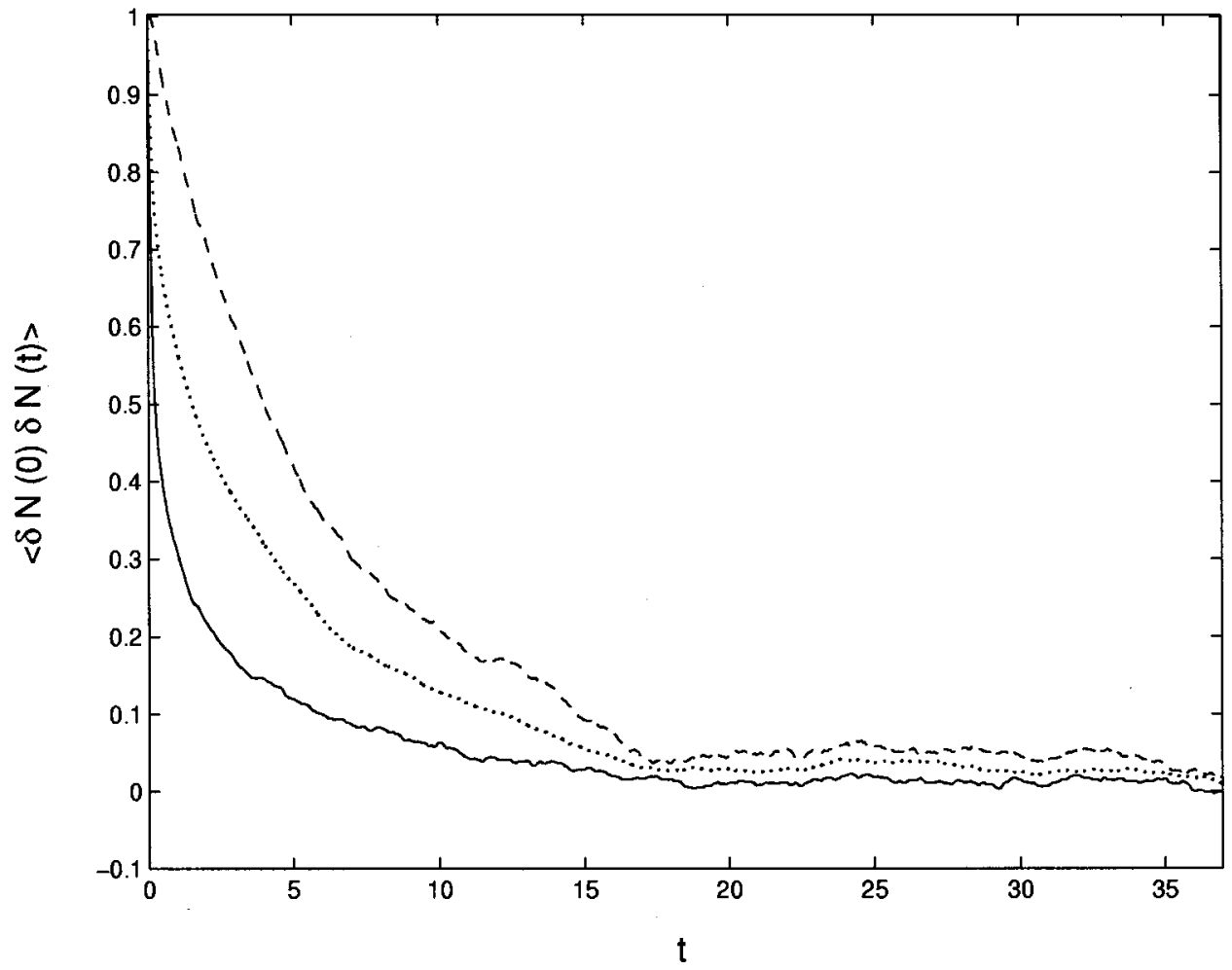

FIG. 8. Similar plot as Fig. 6 but the correlation functions are calculated within a spherical region of radius $R$ $=1.5$. The decay of $A-A$ correlation is shown by the solid line, $B-B$ correlation by the dotted line and that of $A-B$ correlation by the dashed line.

TABLE III. The time constant and the exponent obtained from the stretched exponential fit to the different correlations for $R=1.5$, in the equal size model.

\begin{tabular}{clc}
\hline \hline Constants & \multicolumn{1}{c}{$\tau$} & $\beta$ \\
\hline$A-A$ & 0.42 & 0.35 \\
$B-B$ & 3.68 & 0.67 \\
$A-B$ & 5.5 & 0.81 \\
\hline \hline
\end{tabular}

In order to study the effects of fluctuation in one component on the distribution of the other (that is, their intercorrelations), we have plotted, the normalized joint probability distribution function $P\left(\delta N_{A}, \delta N_{B}\right)$ in Fig. 2. The circles represent the data obtained from the simulation. The bivariate Gaussian distribution fit is also plotted which is represented by the solid lines. It clearly shows that the joint distribution is also nearly Gaussian. The fluctuations in the two compo-

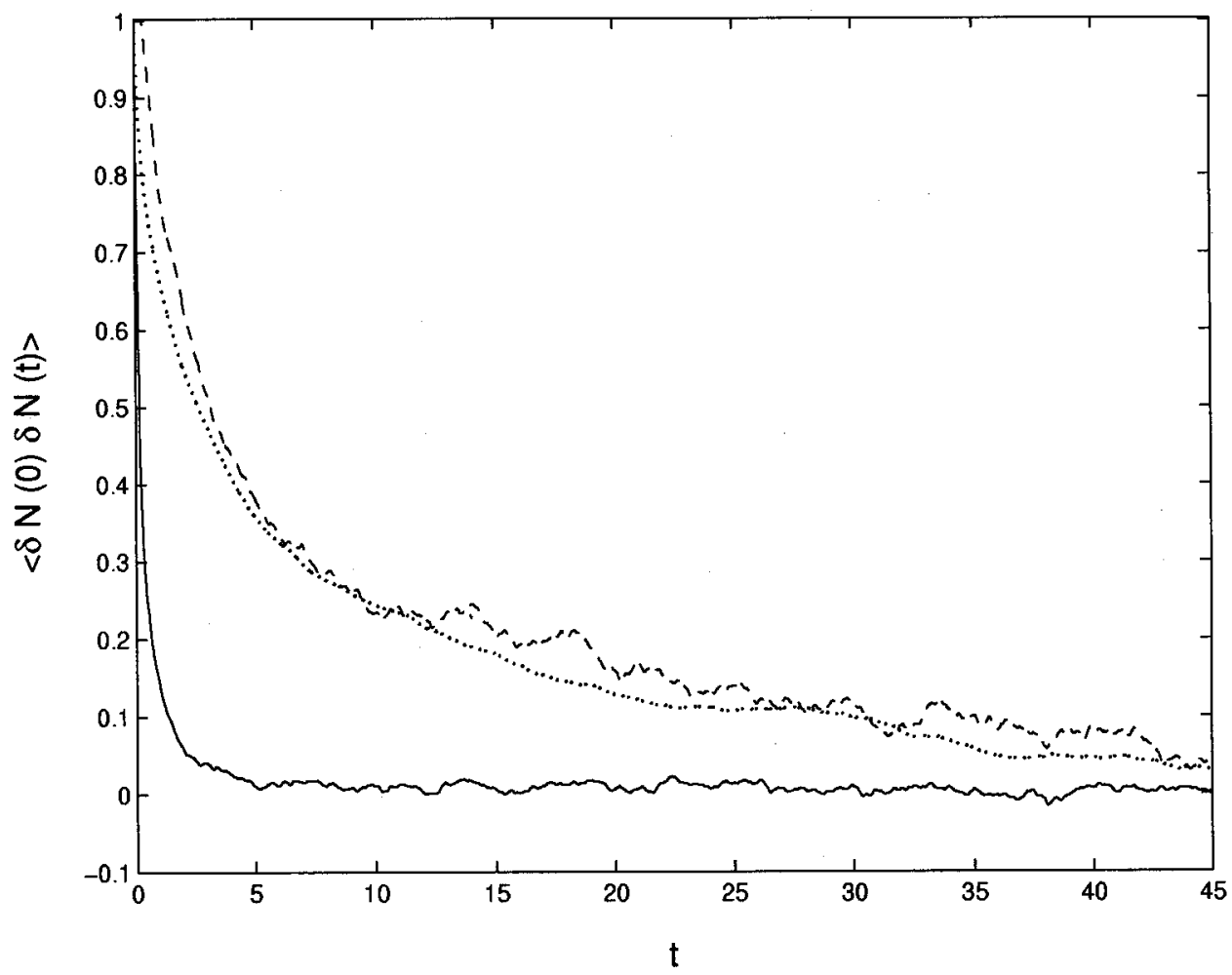

FIG. 9. The normalized time autocorrelation and cross-correlation functions of the composition fluctuations calculated within a spherical region of radius $R=2.0$ for the Kob-Andersen model at $T^{*}=1.0$ and $P^{*}=2.0$. The $A-A, B-B$, and $A-B$ correlation functions are represented, respectively, by the solid, dotted, and dashed lines. Note that the decay of the $A-A$ correlation is very fast compared to the other two correlations. For further detailed discussion, see the text. 
TABLE IV. The time constant and the exponent obtained from the stretched exponential fit to the different correlations for $R=2.0$ at $P^{*}=2.0$, in the Kob-Andersen model.

\begin{tabular}{cll}
\hline \hline Constants & $\tau$ & $\beta$ \\
\hline$A-A$ & 0.19 & 0.47 \\
$B-B$ & 4.3 & 0.48 \\
$A-B$ & 3.0 & 0.41 \\
\hline \hline
\end{tabular}

nents are negatively correlated and this is clearly reflected in the value of the off-diagonal elements of the correlation matrix which is -0.426 . As can be seen from Fig. 2, the negative or positive fluctuation of a given species prefers the counter fluctuation in the other. This shows that in the binary mixture the total number density fluctuation is very small compared to the fluctuation in composition. Such a large fluctuation in composition is obviously expected to modify the properties that are strongly dependent on the composition. Although recently a microscopic explanation for the nonideality in the composition dependence of binary mixtures was presented, ${ }^{1}$ a more detailed theoretical treatment along these directions may prove useful in better understanding the strong nonideal behavior of binary mixtures.

To study the length dependence of composition fluctuation, we have studied fluctuations in a smaller localized volume, of radius $R=1.5$. Figure 3 displays the normalized probability distributions in both the two components. It is evident from this figure that irrespective of the size of the spherical volume the distributions are nearly Gaussian. The width of the individual distributions are again being determined by the composition of the mixture. The average number of components $A$ and $B$ obtained are $\left\langle N_{A}\right\rangle=10.08$ and $\left\langle N_{B}\right\rangle=2.56$, respectively. It is really surprising that fluctua-
TABLE V. The time constant and the exponent obtained from the stretched exponential fit to the different correlations for $R=2.0$ at $P^{*}=4.0$, in the Kob-Andersen model.

\begin{tabular}{clc}
\hline \hline Constants & $\tau$ & $\beta$ \\
\hline$A-A$ & 0.20 & 0.42 \\
$B-B$ & 8.07 & 0.58 \\
$A-B$ & 6.5 & 0.60 \\
\hline \hline
\end{tabular}

tions are Gaussian even when the number of particles involved is so small. The standard deviations are 1.497 and 1.023 , respectively, for the $A$ and $B$ distribution. These results also show that the composition fluctuation is significant in smaller volume even in such a simple model binary mixture. It is found that the joint probability distribution $P\left(\delta N_{A}, \delta N_{B}\right)$ again show the same characteristic feature as was shown in Fig. 2 for larger size spherical volume.

\section{Kob-Andersen (KA) model}

We have mentioned earlier that this model system has been extensively used as a model glass former. In the following we present the simulation results obtained for this model system.

In Fig. 4 we show the normalized probability distributions of the composition fluctuations in both the two components for the spherical volume of radius $R=2.0$ at $P^{*}$ $=2.0$. Here the average reduced density is $\bar{\rho}^{*}=1.018$. The simulation results here again are represented by histograms and the solid lines are pure Gaussians with the same mean and variance. These distributions, as is clearly evident from Fig. 4, are very close to Gaussian. The average number of particles are found to be $\left\langle N_{A}\right\rangle=27.3$ and $\left\langle N_{B}\right\rangle=6.74$. Al-

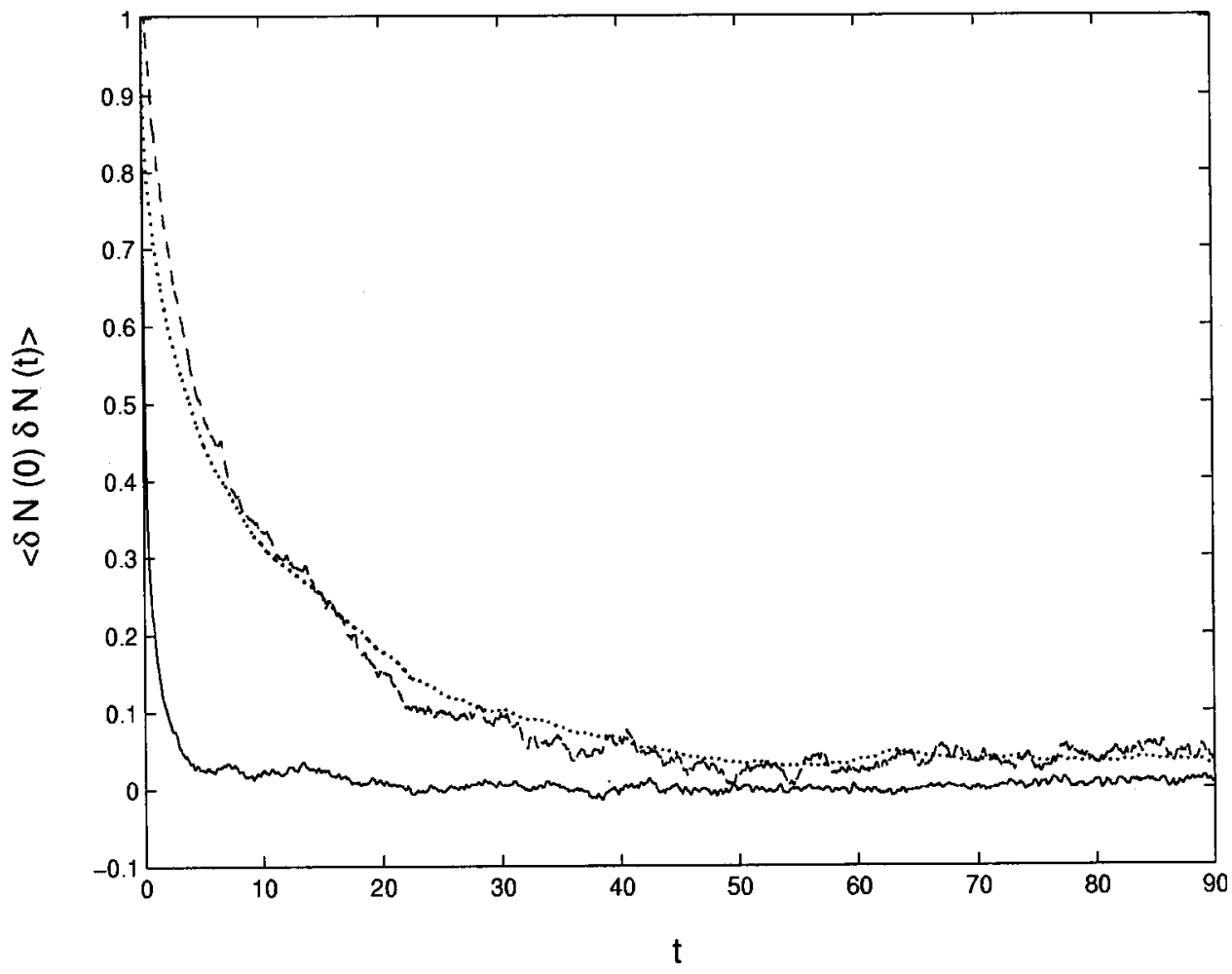

FIG. 10. Similar plot as Fig. 9 but at $T^{*}=1.0$ and $P^{*}=4.0$. The solid line, dotted line, and the dashed line represents the $A-A, B-B$, and $A-B$ correlation functions, respectively. In this case, the decay of all the three correlations have slowed down. For further discussion, see the text. 
though both distributions are nearly Gaussian, it is found that in contrast to the equal size model (model I) the standard deviations for both the distributions are same which is 1.995 . This anomalous behavior can be better explained in terms of the joint probability distribution function, $P\left(\delta N_{A}, \delta N_{B}\right)$. This distribution is plotted in Fig. 5. The circles again represent the simulation results and the solid lines are the bivariate Gaussian distribution fit. One can clearly see from this figure that the joint distribution is nearly Gaussian. The approximate value of the off-diagonal elements of the correlation matrix is -0.203 , which is less than that found for the equal size model. Thus, although the fluctuations in the two components are anticorrelated, the total density fluctuations in the system becomes significant in comparison to the equal size model.

The simulations, as mentioned earlier, are also carried out at relatively high pressure of $P^{*}=4.0$. The average reduced density at this pressure is $\bar{\rho}^{*}=1.091$. The results obtained for $R=2.0$ show that the individual and joint distributions are again be well described by the Gaussian distribution. The average number of particles present in this region are $\left\langle N_{A}\right\rangle=29.2$ and $\left\langle N_{B}\right\rangle=7.45$. Again it is found that the composition fluctuations are quite large and the fluctuations in two different species are negatively correlated in which the value of the off-diagonal elements of the correlation matrix is -0.245 . In the small localized region of radius $R=1.5$, the average number of particles are found to be $\left\langle N_{A}\right\rangle=12.32$ and $\left\langle N_{B}\right\rangle=3.12$. The distributions are again be fit well by the Gaussian distribution, where the spread of the $A$ and $B$ distributions are significantly large, 2.08 and 1.77, respectively. Almost the same value of the correlation is obtained between the fluctuations in $A$ and $B$, as given for the larger volume.

\section{SIMULATION RESULTS: DYNAMICAL CORRELATIONS IN COMPOSITION FLUCTUATION}

The results presented above dealt only the static nature of the composition fluctuations and their intercorrelations. In this section we present the results for the dynamical correlations in composition fluctuation for both the two types of model studied. The pressure dependence of these dynamical response functions, which has been carried out in the KobAndersen (KA) model, is also presented.

\section{A. Equal size model (model I)}

In Fig. 6, we show the decay profiles of the time autocorrelation and cross-correlation functions of the composition fluctuation in a spherical volume of radius $R=2.0$. It clearly shows that the decay of cross-correlation function is slower than both the autocorrelation functions. As the interaction strength between the unlike components is the strongest (see Table I), $A$ and $B$ molecules are preferred to remain surrounded by the opposite species. Due to this strong caging effect, the decay of $\left\langle\delta N_{A}(0) \delta N_{B}(t)\right\rangle$ becomes slow. The relaxation of these dynamical correlations are significantly nonexponential and a slow tail appears in the long time which can be seen clearly in the Fig. 6. To get an estimate of the degree of nonexponentiality, we have fitted these functions both to multiexponential function (of order 3 ) and also to stretched exponential function, $A \exp (-t / \tau)^{\beta}$. We find that the fitting to the stretched exponential gives more accurate description. In Fig. 7, we plot these correlation functions along with the stretched exponential fit. The values of the time constants $(\tau)$ and the corresponding exponents $(\beta)$ are given in Table II. These values clearly indicate that the decay of the cross-correlation function is slower than that of the corresponding autocorrelation functions and is less nonexponential. As the number of $B$ molecules present is much less, almost all of them are caged by $A$ molecules. However, there are always some excess $A$ molecules near the surface of the localized region which are relatively free. Exchange of these $A$ molecules with available $B$ molecules is energetically favorable. This could be attributed to the relatively faster decay of $A-A$ correlations.

In order to see how the dynamical correlations get effected for smaller volumes, we have plotted in Fig. 8 all the three time correlation functions calculated for the spherical volume of radius $R=1.5$. Table III provide the values of the time constants and the exponents obtained from the stretched exponential fit. It is clearly seen from Fig. 8 that although the initial decay of these correlations are faster compared to larger volumes, a slower tail appears in the longer time. The suppression of long wave length fluctuations due to insufficient size of the spherical volume could be the microscopic origin of this very slow decay.

\section{B. Kob-Andersen (KA) model}

For this model system the decay profiles of the time correlation functions are displayed in Fig. 9 for the spherical volume of radius $R=2.0$ at $P^{*}=2.0$. Most interestingly, it is clearly evident while the decay of $A-A$ correlation is fast (as in model I), the $B-B$ correlation decays more or less in a similar fashion as that of $A-B$ correlation. This behavior is also reflected in the values of their time constants and the respective exponents which are obtained from the stretched exponential fit. The fitting parameters are presented in Table IV. One should remember at this point that in this model the sizes of $A$ and $B$ are different (see Table I). The size of the $A$ molecules is bigger than that of $B$ molecules which is larger than the mutual size of $A$ and $B$ molecules. This model also considers the $A-B$ interaction being the strongest (see Table I). As the fraction of $B$ present is 0.2 and the $A-B$ interaction is very strong, most of the $B$ molecules are surrounded by the cage of $A$ molecules. However, there could be significant number of $A$ molecules specifically near the surface of the spherical volume which are free of any cage formed by the opposite species. In addition to the strong $A-B$ interaction, the movement of these relatively free $A$ molecules out of the spherical volume is driven by the smaller size of the $B$ molecules and also by the small mutual size of the molecules.

Figure 10 present how the decay of these correlations in a region of radius $R=2.0$, get modified at high pressure $P^{*}=4.0$. As expected the correlation functions show a slower decay pattern and are again non-exponential. The different time constants and the exponents again obtained from the stretched exponential fit are given in Table V. 


\section{CONCLUSION}

In this paper we have presented isothermal-isobaric (NPT) ensemble molecular dynamics simulation study of the composition fluctuations in nonphase separating dense Lennard-Jones binary mixtures of two types, in both of which the interaction between the dissimilar species is more favored. One of the model binary mixtures (known as KobAndersen model) is a well-known glass former. It is apparent from our simulations that irrespective of the size of the spherical volumes considered, the composition fluctuations and their intercorrelations are well described by Gaussian statistics. The standard deviation of the distributions is found to be quite large, indicating broad distributions. The equilibrium spontaneous fluctuations in the two components are shown to be negatively correlated, indicating that the occurance of total density fluctuations in these local volumes is very small.

The relative abundance of the composition fluctuation indicates presence of many different compositions within small volumes. Thus, the system is indeed locally heterogeneous. It will be a worthwhile exercise to find the distribution of relaxation times of these regions. However, this is meaningful only when there is a broad spread in the relaxation times, expected in supercooled liquid. We are currently investigating this problem.

We found that the time correlations of fluctuations in $N_{A}$ and $N_{B}$ show nonexponential dynamics. As expected, the cross-correlation decay is slowest because in the binary mixtures of composition $N_{B} / N=0.2$, most of the $B$ molecules are surrounded by $A$ molecules. This result clearly suggests that the most slowly relaxing local configurations should involve $B$ molecules surrounded by $A$ molecules-that is, maximize the number of $A-B$ bonds. Recently, Glotzer et al. ${ }^{6}$ have explored the dynamics in the KA model along this line. Further work in this area is warranted.

\section{ACKNOWLEDGMENTS}

We thank Dr. Sarika Bhattacharyya and Goundla Srinivas for many helpful discussions and suggestions. We also thank Dr. S. Balasubramanian (JNCASR) for his help in do- ing the simulations. This work was supported in part by the Council of Scientific and Industrial Research (CSIR), India and the Department of Science and Technology (DST), India. One of the authors (R.K.M) thanks the University Grants Commission (UGC) for providing the Research Scholarship.

${ }^{1}$ G. Srinivas, A. Mukherjee, and B. Bagchi, J. Chem. Phys. 114, 6220 (2001).

${ }^{2}$ C. A. Angell, Science 267, 1924 (1995); M. D. Ediger, C. A. Angell, and S. R. Nagel, J. Phys. Chem. 100, 13200 (1996).

${ }^{3}$ M. T. Cicerone, F. R. Blackburn, and M. D. Ediger, J. Chem. Phys. 102, 471 (1995); M. T. Cicerone and M. D. Ediger, ibid. 103, 5684 (1995); U. Tracht, M. Wilhelm, A. Heuer, H. Feng, K. Schmidt-Rohr, and H. W. Spiess, Phys. Rev. Lett. 81, 2727 (1998).

${ }^{4}$ W. Kob, C. Donati, S. J. Plimpton, P. H. Poole, and S. C. Glotzer, Phys. Rev. Lett. 79, 2827 (1997); C. Donati, J. F. Douglas, W. Kob, S. J. Plimpton, P. H. Poole, and S. C. Glotzer, ibid. 80, 2338 (1998).

${ }^{5}$ W. Kob and H. C. Andersen, Phys. Rev. Lett. 73, 1376 (1994); Phys. Rev. E 51, 4626 (1995).

${ }^{6}$ C. Donati, S. C. Glotzer, P. H. Poole, W. Kob, and S. J. Plimpton, Phys. Rev. E 60, 3107 (1999).

${ }^{7}$ R. K. Murarka and B. Bagchi, J. Chem. Phys. 115, 5513 (2001).

${ }^{8}$ G. E. Crooks and D. Chandler, Phys. Rev. E 56, 4217 (1997).

${ }^{9}$ D. M. Huang and D. Chandler, Phys. Rev. E 61, 1501 (2000).

${ }^{10}$ T. M. Turskett, S. Torquato, and P. G. Debenedetti, Phys. Rev. E 58, 7369 (1998).

${ }^{11}$ G. Hummer, S. Garde, A. E. Garcia, A. Pohorille, and L. R. Pratt, Proc. Natl. Acad. Sci. U.S.A. 93, 8951 (1996); G. Hummer, S. Garde, A. E. Garcia, M. E. Paulaitis, and L. R. Pratt, J. Phys. Chem. B 102, 10469 (1998).

${ }^{12}$ S. Sastry, P. G. Debenedetti, and F. H. Stillinger, Nature (London) 393, 554 (1998).

${ }^{13}$ T. A. Weber and F. H. Stillinger, Phys. Rev. B 31, 1954 (1985).

${ }^{14}$ J. P. Hansen and I. R. McDonald, Theory of Simple Liquids (Academic, New York, 1986).

${ }^{15}$ D. Frenkel and B. Smit, Understanding Molecular Simulation: From Algorithm to Applications (Academic, San Diego, 1996).

${ }^{16}$ G. J. Martyna, D. J. Tobias, and M. L. Klein, J. Chem. Phys. 101, 4177 (1994); H. C. Andersen, ibid. 72, 2384 (1980); S. Nose, Mol. Phys. 52, 255 (1984); W. G. Hoover, Phys. Rev. A 31, 1695 (1985).

${ }^{17}$ G. J. Martyna, M. E. Tuckerman, and M. L. Klein, J. Chem. Phys. 97, 2635 (1992).

${ }^{18}$ M. E. Tuckerman, G. J. Martyna, and B. J. Berne, J. Chem. Phys. 97, 1990 (1992).

${ }^{19}$ H. Yoshida, Phys. Lett. A 150, 260 (1990); M. Suzuki, J. Math. Phys. 32, 400 (1991).

${ }^{20}$ G. J. Martyna, M. E. Tuckerman, D. J. Tobias, and M. L. Klein, Mol. Phys. 87, 1117 (1996). 